\title{
In vivo evaluation of anticryptosporidiosis activity of the methanolic extract of the plant Umbilicus rupestris
}

\author{
AFAF BENHOUDA", DJAHIDA BENHOUDA, MASSINISSA YAHIA \\ Biotechnology's Laboratory of the Bioactive Molecules and the Cellular Physiopathology, Faculty of Biological Sciences, Department of Biology and \\ Living Organisms, University of Mustapha Ben Boulaid. 53 Constantine Rd, Fésdis, Batna 05078, Algeria. Tel: +213-676-002083. \\ vemail: a.benhouda@univ-batna2.dz
}

Manuscript received: 18 October 2019. Revision accepted: 5 November 2019.

\begin{abstract}
Benhouda A, Benhouda D, Yahia M. 2019. In vivo evaluation of anticryptosporidiosis activity of the methanolic extract of the plant Umbilicus rupestris. Biodiversitas 20: 3478-3483. Umbilicus rupestris (Crassulaceae) is a medicinal plant used in general traditional medicine to cure inflammation and irritation of the skin. The present research is aimed to evaluate the antiparasitic activity of the methanolic extract of the plant $\mathrm{URMeOH}$ of $U$. rupestris against the Cryptosporidium infection in immunocompetent and immunosuppressed rats experimentally infected. Twenty-one female rats were divided into two groups. Control group (group I) and experimental group (Group II). The group I was further divided into three equal groups (normal group infected and immunosuppressed infected group). The experimental group was divided into two immunosuppressed and four equal groups and two immunocompetent infected. Cryptosporidium oocysts were isolated from bovine species stools and used to infect rats. Experimental subgroups received $\mathrm{URMeOH}$ two as dose $100 \mathrm{mg} / \mathrm{kg}$ b.w. and $200 \mathrm{mg} / \mathrm{kg}$ b.w. and continued until 15 days. Two weeks after the administration of $\mathrm{URMeOH}$, feces of rats were examined for the detection of Cryptosporidium oocysts by Ziehl-Neelsen and immunofluorescence techniques, the animals were sacrificed; their small intestines were processed and examined for the detection of pathological lesions after histopathological study. In addition, the activity of myeloperoxidase (MPO) was measured in sections of the jejunum. Concerned the results, we observed a statistically significant $(\mathrm{P}<0.001)$ increase in the number of oocysts of Cryptosporidium in the stool for sub infected immunosuppressed groups and an increase of MPO activity compared to the corresponding subgroups immunocompetent subgroups. The URMeOH could remove Cryptosporidium oocysts from feces and intestinal sections subgroup infected immunocompetent rats receiving URMeOH. Moreover, the oocysts were significantly reduced in all other subgroups experimental infected compared to infected control subgroups. Intestinal sections in all subgroups received URMeOH revealed a more or less normal architecture. In addition, the reduction of MPO activity level was also detected in all experimental subgroups.
\end{abstract}

Keywords: Algeria, Cryptosporidium, Umbilicus rupestris, immunofluorescence, MPO, oocysts

\section{INTRODUCTION}

Medicinal plants are important in pharmacological research and drug development, not only when plant constituents are used directly as therapeutic agents, but also as raw materials for drug synthesis or as models for pharmacologically active compounds (Atanasov 2015).

Cryptosporidiosis is a parasitic disease caused by Cryptosporidium spp., a parasite in the phylum Apicomplexa. Despite not being identified until 1976, it is one of the most common water-borne diseases and is found worldwide (Bouzid et al.2013). This affects the mammalian intestine and is typically a short-term acute infection. It is spread through the fecal-oral route, often through contaminated water. The parasite is transmitted by the oocysts once ingested; it exists in the small intestine which translates into an infection of the intestinal epithelial tissue (Di Genova and Tonelli 2016). The main symptom is diarrhea in people whose immune system is intact. In immunosuppressed individuals, such as AIDS patients, the symptoms are particularly in the form of severe dehydration, onset of electrolyte imbalances, malnutrition, and possibly death and also diarrhea (Leitch et He 2011).
For treatment against this parasite, some antiparasitic agents such as paromomycin; nitazoxanide and azithromycin are used, but usually have temporary effects. The search for anticryptosporidiosis plants is very interesting at the moment (Sparks et al. 2015).

Umbilicus rupestris (Salisb.) Dandy is a hardy perennial plant, belongs to the family of Crassulaceae. It presents at the level of rocks, cliffs, and the old walls, on the coasts and in mountains; only in North Africa (Gonzalez-Tejero et al.2008). Leaves of this plant are used in traditional medicine against the ignitions of skin, wounds, like cataplasm, against burns disinfectant, parasiticidal, healing. Infused (leaves) of this plant is used like an ophthalmic disinfectant (Benhouda et al.2014).

This work aim is to study the antiparasitic activity of the methanolic extract of $U$. rupestris. This plant contains differents secondary metabolites and according to Benhouda et al. (2014), the methanolic extract of this plant has an effect on all the following bacterial strains $S$. aureus ATCC 25923, E. coli ATCC 25922 and P. aeruginosa ATCC 27853. 


\section{MATERIALS AND METHODS}

\section{Parasites}

Fecal samples were collected in sterile boxes of 60 calves with an age (1 day up to 6 months) from different farms (Djerma, Ain Yagout, Chemerra in the months of April and May 2016).

\section{Detection of the Cryptosporidium parasite \\ Concentration by the RITCHI technique}

This technique increases the sensitivity of the search for cystic forms or eggs. After the collection of samples we transferred our samples in our laboratory LBMBPC and are stored at $4{ }^{\circ} \mathrm{C}$. We performed Ritchie's method for sample concentration according to the method of Coelho et al (2009), we diluted one volume of each sample in 10 volumes of Ritchie reagent $(100 \mathrm{~mL}$ of formalin, $9 \mathrm{~g}$ of $\mathrm{NaCl}, 900 \mathrm{~mL}$ of distilled water), after mixing for a few seconds, then $1 / 3$ of ether were added to $2 / 3$ of the mixture and then the tube was placed in the centrifuge at 1500 $\mathrm{rpm} / \mathrm{min}$, after centrifugation the supernatant was discarded and a direct examination was carried out on the pellet.

\section{Ziehl-Neelsen (MZN) staining technique}

This staining technique makes it possible to observe the oocysts of Cryptosporidium which are colored in pink on a blue background. After having recovered the Ritchie pellet, the pellet was spread on a slide and dried in the open air, then fixed with methanol for $3 \mathrm{~min}$, dried and then stained with fushine for $30 \mathrm{~min}$, washing then colored water with a solution of methylene blue for $3 \mathrm{~min}$, after washing with water and dried in the open air, finally observed under a microscope at magnification 400 (objective 100) or magnification 1000 (objective 100) (Williams 1998).

\section{Direct immunofluorescence detection technique}

This technique is more specific for the detection of the Cryptosporidium parasite, we took $50 \mu \mathrm{l}$ of our concentrated stool, each slide contains three wells one for the sample and the other two for the positive and negative control. After spreading the sample on the well, we allowed to dry at $42^{\circ} \mathrm{C}$, then a drop of the solution of the monoclonal antibodies anti-Cryptosporidium and Giardia containing a stabilizing protein and $0.1 \%$ of sodium azide then a drop of dye Fluorescent was added which is the solution of Eriochrome Black after these slides were kept in a humidity chamber, after $45 \mathrm{~min}$ the wells are washed with $20 \mathrm{X}$ wash buffer concentrate. After washing, a mounting solution which is the glycerol buffer containing formalin, an antiquencher and $0.05 \%$ sodium azide is applied was applied to all wells for observation on a fluorescence microscope equipped with a filter system for fluorescein isothiocyanate (FITC) with some characteristics: Excitation 490-500 nm, filter barrier: 510$530 \mathrm{~nm}$. Knowing that the formalin positive control is a stool preparation containing Cryptosporidium and Giardia oocysts containing $0.09 \%$ thimerosal and the negative control are formalinized stools containing 0.09\% thimerosal, these controls have undergone the same procedure.

\section{Culture of the parasite Cryptosporidium}

Cryptosporidium parasites are isolated from the feces of cattle infected with Cryptosporidium. The stools of cattle are homogenized in an equal volume of water and filtered with a filter of $455 \mu \mathrm{m}$. The fat is removed by sedimentation using petroleum ether. $1 \mathrm{~mL}$ of the petroleum ether is mixed with $9 \mathrm{~mL}$ of each homogenate and centrifuged at $2000 \mathrm{rpm} / \mathrm{min}$ and the supernatant with the fat is removed (Lumb et al. 1998). After the homogenous was left for a quarter of an hour. The last layer contains most of the oocysts was recovered and centrifuged at $2000 \mathrm{rpm}$ for $10 \mathrm{~min}$. The bottom is suspended with sodium chloride $(\mathrm{NaCl})$ and after centrifugation at $2000 \mathrm{rpm}$ for $10 \mathrm{~min}$ and the oocysts are isolated from the last layer of the supernatant. The oocysts are washed three times with water and stored in potassium dichromate at $4^{\circ} \mathrm{C}$. After being washed four times with the solution of Hanks balanced salt before their use. The excitation of the sporozoites is completed by incubating the oocysts at $37^{\circ} \mathrm{C}$ with $0.5 \%$ trypsin in the Hanks balanced salt solution for $30 \mathrm{~min}$, after being washed in RPMI medium, and incubated in $0.4 \%$ of serum fetal bovine at 37 ${ }^{\circ} \mathrm{C}$ for $45 \mathrm{~min}$. After the mixture was washed with RMPI medium and then centrifuged at $2000 \mathrm{rpm}$ for $10 \mathrm{~min}$ and the parasites are found in the last layer (Gaafar 2007).

\section{The antiparasitic activity against Cryptosporidium- induced diarrhea \\ Collection of plant material}

The leaves of $U$. rupestris were collected from their natural habitat around Tibhirine, Batna. This plant was identified by Dr.OUDJHIH, Laboratory of Botanic, Department of Agronomy, BATNA-1-, ALGERIA. The leaves were dried under shade for 40 days at room temperature, dried leaves parts were blended into fine powder and stored in the dark at a dry place.

\section{Preparation of plant extract}

$1 \mathrm{Kg}$ of powdered leaves was extracted with petroleum ether three times $5 \mathrm{~L}$ for each time. Then, the marc was dried and extracted with chloroform three times $5 \mathrm{~L}$ for each time and with methanol three times $5 \mathrm{~L}$ for each time and the supernatants were filtered sequentially using cloth filter, cotton wool, and Whatman filter paper. The solvents were then evaporated under reduced pressure (204 mbar) and controlled temperature $\left(30^{\circ} \mathrm{C}\right)$ using a vacuum rotary evaporator (Buchi Rotavapor).

\section{Experimental animals}

Wistar albino rats for either sex (140-170 g) procured for Research institute of both sexes were housed separately in plastic cage at temperature of $(23 \pm 2){ }^{\circ} \mathrm{C}$ and $50-55 \%$ relative humidity, with a 12 light/dark cycle respectively before and during the experiment. Animals were allowed access to standard pellet diet and water ad libitum. The protocol of the experiment (DEC-DGK number: 2008.I.01.004).

The experiment was carried out according to the method previously described by Gaafar (2007). The rats were divided into two groups. Control group (Group I) and 
experimental group (Group II).

Control group I ( 9 rats) was subdivided into three equal subgroups (3 rats each). (i) Subgroup I a: normal group, served as a control. (ii) Subgroup I b: infected group with Cryptosporidium oocysts isolated at a dose of $10^{4}$ oocysts/rat. (iii) Subgroup I c: an immunosuppressedinfected group, this group is rendered immunosuppressed after injection of cyclophosphamide with a dose of 17 $\mathrm{mg} / \mathrm{kg}$ p.c. intraperitoneally one week before infection with the parasite. The dose of the isolated Cryptosporidium oocyst infection is 104 oocyst/rat.

The experimental group (12 rats); was subdivided into four equal subgroups (3 rats each). (i) Subgroup IIa: Group infected with the parasite, after infection the group was treated with URMeOH extract $(100 \mathrm{mg} / \mathrm{kg}$ b.w.) for two weeks. The dose of isolated Cryptosporidium oocysts is $10^{4}$ oocyst/rat. (ii) Subgroup IIb: an immunosuppressedinfected group; this group is immunosuppressed by the injection of cyclophosphamide with a single dose of 17 $\mathrm{mg} / \mathrm{kg}$ b.w. a week before then infected with the parasite after this subgroup was treated with URMeOH $(100 \mathrm{mg} / \mathrm{kg}$ b.w.) for two weeks. (iii) Subgroup II c: an infected group, this group is infected and then treated with URMeOH (200 $\mathrm{mg} / \mathrm{kg}$ b.w.) for a period of 2 weeks. The dose of infection is $10^{4}$ oocyst/rat. (iv) Subgroup II d: an immunosuppressed-infected group, this group is immunosuppressed with cyclophosphamide $(17 \mathrm{mg} / \mathrm{kg} \quad$ b.w. $)$ by the intraperitoneal route administration. After one week of infection, this group is infected with the parasite and then treated with URMOH $(200 \mathrm{mg} / \mathrm{kg}$ b.w.) for 2 weeks. These concentrations are used after testing the acute and subacute toxicity according to Benhouda et al. (2015)

\section{Evaluation of the efficacy of Umbilicus rupestris extract}

Survival of rats. Survival rate of both immunocompetent and immunosuppressed subgroups of rats were evaluated.

Analysis of fecal samples. Undiluted stool samples from rats from all subgroups were collected on the last day of the experiment and then stained with MZN and examined with microscopy $(\mathrm{x} 100)$ for the presence or absence of oocysts of Cryptosporidium.

\section{MPO activity}

Myeloperoxidase activity (MPO activity) was measured in extracts of full-thickness sections (200-300 mg) of rats jejunum in all subgroups at the day of sacrifice. Tissue samples were weighed and homogenized with hexadecyltrimethylammonium bromide (HTAB) buffer $(0.5 \%$ HTAB in $50 \mathrm{mM}$ phosphate buffer, $\mathrm{pH} 6.0,4^{\circ} \mathrm{C}$ ).

The homogenates were freeze-thawed three times and then centrifuged at $35,000 \mathrm{~g}$ for $30 \mathrm{~min}$. The pellets were removed, and the supernatants were assayed for soluble protein and for MPO activity. MPO activity was measured by adding $0.1 \mathrm{~mL}$ supernatant to $2.9 \mathrm{~mol}$ reaction buffer (50 mM phosphate buffer, $\mathrm{pH} 6.0$, containing $0.167 \mathrm{mg} / \mathrm{mL}$ o-dianisidine hydrochloride and $0.0005 \%$ hydrogen peroxide). After $1 \mathrm{~min}$, the change in absorbency at $460 \mathrm{~nm}$ was measured by the spectrophotometer. One unit of MPO activity was defined as that degrading $1 \mathrm{lmol}$ of peroxidase per minute at $25^{\circ} \mathrm{C}$. The MPO activity was expressed per $\mathrm{mg}$ of protein. Soluble protein in the tissue supernatant was assayed using Lowry's method.

\section{RESULTS AND DISCUSSION}

Diarrhea is considered a very serious management problem in immunosuppressed patients, as well as in normal people in developed and developing countries. Pathogenic intestinal protozoa are the main causes of this diarrhea, among which Cryptosporidium produces outbreaks that occur regularly throughout the world (Farthing 2000).

Cryptosporidiosis is a parasitic disease caused by Cryptosporidium despite was not identified until 1976, it is one of the most common waterborne diseases and is found worldwide. It affects mammalian gut and is usually an acute short-term infection. It is spread by the fecal-oral route, often by contaminated water (Sponseller et al. 2014). The parasite is transmitted by oocysts found in the environment, once ingested it migrates to the small intestine causing infection of the intestinal epithelial tissue (Bouzid et al.2013). The antiparasitic effect of extract depends on secondary metabolites (tannins, flavonoids, and phenols) which have different activities: anti-oxidant, antiinflammatory (Gaafar 2012).

Phenolic compounds are secondary metabolites that play an important role in antiparasitic activity. It has been shown that the decrease in larval migration by extracts of plants could be the cause of the disruption of their installation in the lining of the gastrointestinal mucosa. This inhibitory action of parasite larval migration would be made possible by the large families of chemical compounds identified in this study, namely tannins, flavonoids and total phenols (Molan et al. 2003). We suggested that blocking lipid synthesis may be an important component of the antimicrobial activity of $\mathrm{URMeOH}$ (Adetumbi et al.1986).

\section{Survival rate of rats}

The number of dead animals was recorded in the period of our study, one spleen in the infected immunosuppressed subgroup and another immunocompetent of the infected subgroup. In addition, all rats are alive until the end of the study. Thus, the survival rate recorded in this study was $90.47 \%$ (the number of rats remaining $\mathrm{x} 100 /$ the total number of rats) for the two immunocompetent and immunosuppressed groups of the rats.

In this study, immunosuppression of experimental animals was induced by cyclophosphamide (Endoxan) at a dose of $17 \mathrm{mg} / \mathrm{kg}$ b.w The choice of this drug was made because of its ability to immunosuppress both humoral and cell-mediated. Intraperitoneal injection of cyclophosphamide with the given dose may produce little or no death in normal rats. The cause of the rat death in immunosuppresses subgroup certainly is related to the situation of immunosuppression. In contrast, the cause of immunocompetent rat death in the subgroup is still not apparent (Camenga et al. 1974). 


\section{Results of fecal samples}

After the recovery of the stool in sterile boxes and analysis by Zhiels Neelseen technique, we obtained the results that are presented in (Figures 1 and 2). In the control group infected with Cryptosporidium, the oocysts are better visualized by MZN, dark pink spots are observed, on a blue-green background. The observation was made with the objective 40 then 100, using a drop of oil immersion.

URMeOH extract successfully eradicated Cryptosporidium oocysts in the feces of the infected subgroup, we observed a decrease of oocysts in all the experimental subgroups by contribution to the infected control subgroups. There was an increase in the number of Cryptosporidium oocysts in the stool samples of the immunosuppressed subgroups infected with immunocompetent subgroups. No parasite was detected in normal subgroup stool samples.

The detection of Cryptosporidium oocysts in feces is based on staining, concentration (sedimentation or flotation) or immuno-labeling techniques. Staining techniques include Ziehl-Neelsen staining, auraminephenol staining and Heine-negative staining (Casemore 1991). The advantages of these techniques are their low cost and their speed of implementation while the disadvantages reside in the need for specific equipment such as the use of the fluorescence or phase contrast microscope for the auramine-phenol and Heine stains, respectively. In addition, Ziehl-Neelsen staining is often considered the reference technique, may be accompanied by a number of false positives (yeasts, fungi or bacteria spores) although the size of the elements "Pseudocryptosporidians" theoretically avoids confusion (Casemore 1991). Sensitivity defects, due to lack of staining of certain oocysts, have also been reported, particularly with acid-fast stains such as Ziehl-Neelsen and Kinyoun (Somoskövi et al. 2001).

Among the techniques used to detect Cryptosporidium in fecal samples, the direct immunofluorescence assay (FID) is the most important test (Fayer et al. 2000). This method has good sensitivity and high specificity than traditional staining techniques. We tested our fecal specimens by the direct immunofluorescence technique to confirm the results of the Zhiels Neelseen technique. We found that in the control group infected with Cryptosporidium the oocysts are better visualized with immunofluorescence contents which appear with the green color on a black background (Figure 3). We also observed a decrease of oocysts in all the experimental subgroups by contribution to the infected control subgroups and an increase in number of Cryptosporidium oocysts in the stool samples of the infected immunosuppressed subgroups compared to the immunocompetent subgroups. which confirms the results of the technique Zhiel Nielsen.
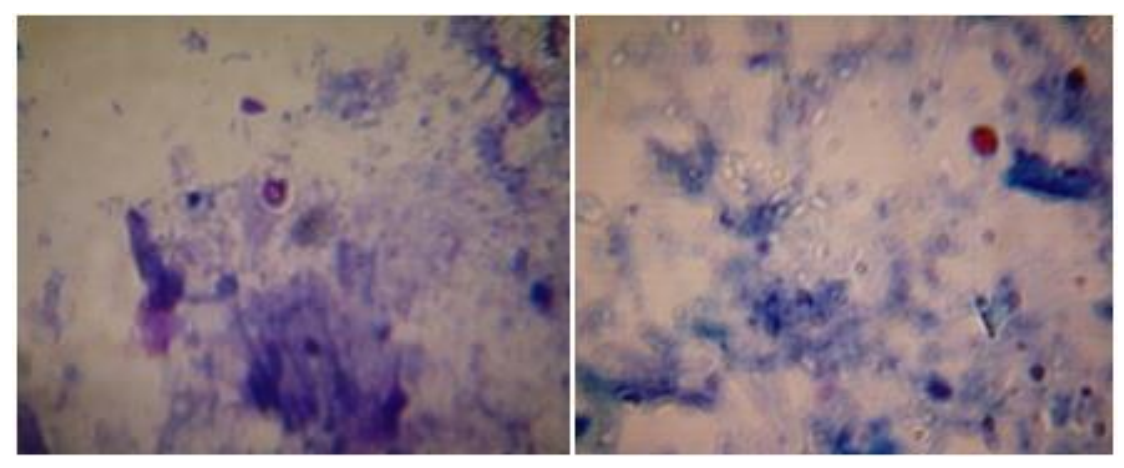

Figure 1. Zhiels Neelseen results from Cryptosporidium infected cattle
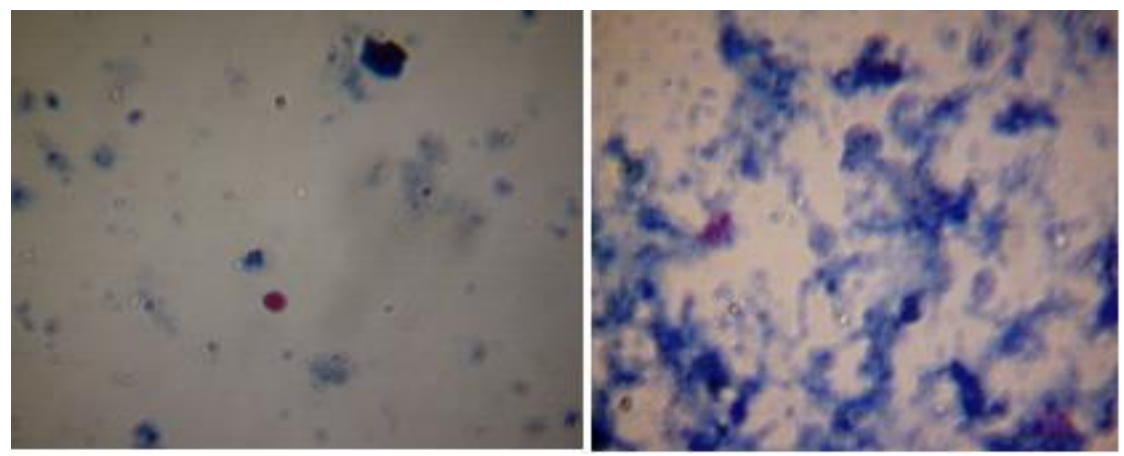

Figure 2. Zhiels Neelseen results from Cryptosporidium infected rats 


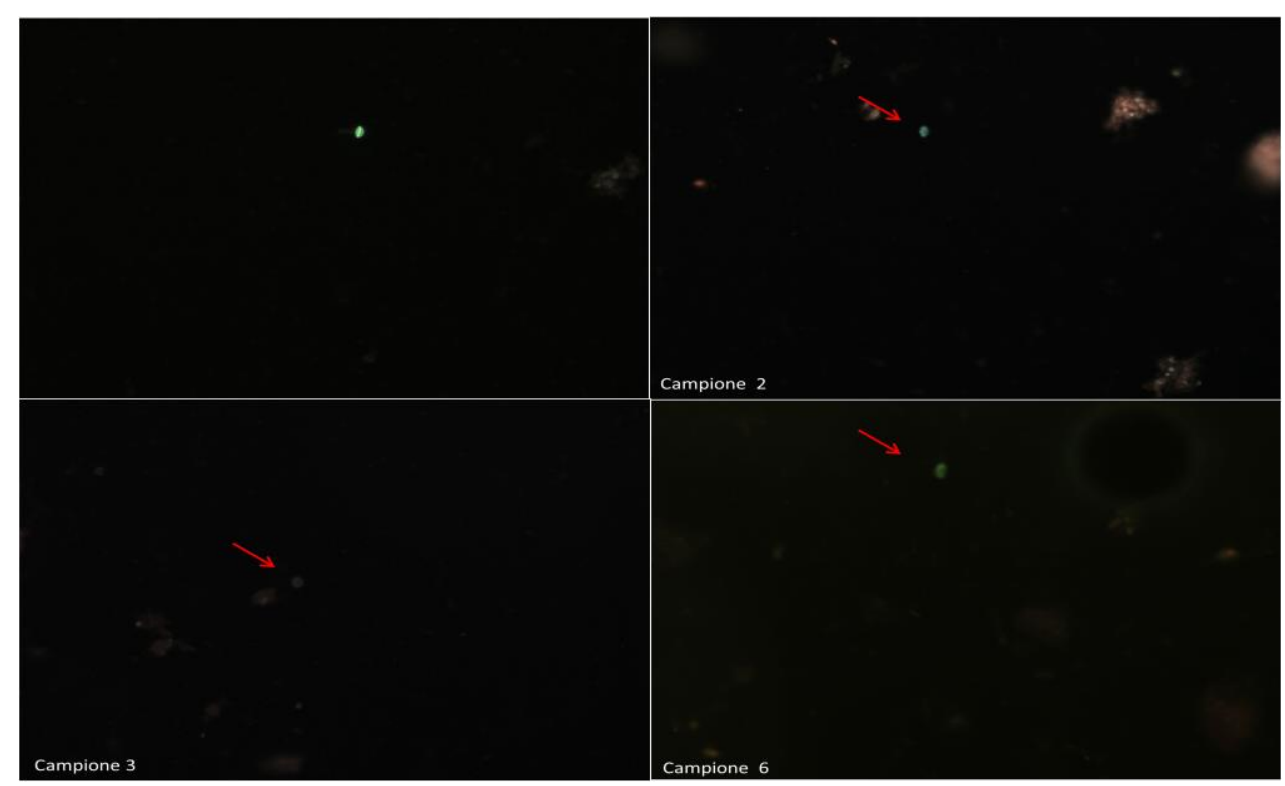

Figure 3. Cryptosporidium detection with direct immunofluorescence

\section{Results of MPO}

From the results obtained, we found that parasite infection caused an increase in MPO activity in the immunosuppressed group infected with a value of 19.78 $\mu / \mathrm{mg}$ which is highly significantly higher $(\mathrm{P} \leq 0.001)$ to the control $14.23 \mu / \mathrm{mg}$. The extract with the concentration 200 $\mathrm{mg} / \mathrm{kg}$ b.w. presented the lower the activity of the enzyme to $12 \mu / \mathrm{mg}$ without significant difference compared to the groups treated with the doses 100 and $200 \mathrm{mg} / \mathrm{Kg}$ b.w. (P $>0.05)$.

For the immunocompetent rats, the infection increased the activity of the enzyme with a significant difference ( $P$ $\leq 0.05$ ) still by contribution to the control group (Table 1 ).

Myeloperoxidase (MPO) is a heme enzyme present in high concentrations ( $\pm 5 \%$ by weight) in the primary granules of polymorphonuclear neutrophilic cells (Serteyn et al.2003).

Myeloperoxidase (MPO) is part of the peroxidase subfamily. It is expressed most abundantly in immune cells such as leukocytes, neutrophils and (Khan et al. 2014; Lieu et al. 2015) lymphocytes and macrophages (Nicholls et Hazen. 2005).

Neutrophils play an important role in innate immunity and defense against microbial agents (Klebanoff 2005). MPO and neutrophils contain several enzymes that have antimicrobial properties, such as defensins, serine proteases, cathepsin G, alkaline phosphatase, lysozyme, collagenase, lactoferrin and cathepsin (Yamaguchi et al. 2015; Khan et al. 2018).

Serteyn et al. (2003) mentioned that when infection occurs in the body, neutrophils are the first cells in the immune system that are activated to fight infection. They reach the site of infection by the phenomenon of chiomiotactism and diapedesis (through increased vascular permeability) and then recognize and phagocytose bacteria and other microorganisms in the phagosome.

High levels of MPO in body fluids and tissues indicate significant or excessive activation of neutrophils. In recent years, MPO has been used as a marker of the intensity of inflammation in many pathologies (Serteyn et al.2003).

Table 1. Values of MPO in different subgroups in Wistar rats

\begin{tabular}{ll}
\hline Subgroups of animal & Values of myeloperoxidase $(\boldsymbol{\mu} / \mathbf{m g}$ of protein) \\
\hline Group I : Immunocompetent group & 5.06 \\
No infected control & $9.45^{* *}$ \\
Infected immunocompetent & 6.45 \\
Immunocompetent treated with URMeOH $100 \mathrm{mg} / \mathrm{Kg} \mathrm{b.w.}$ & 6.25 \\
Immunocompetent treated with URMeOH $200 \mathrm{mg} / \mathrm{Kg}$ b.w. & \\
Group II : Immunosuppressed group & 14.23 \\
Immunosuppressed control & $19.78^{* * *}$ \\
Immunosuppressed infected & $12.12^{*}$ \\
Immunosuppressed treated with URMeOH $100 \mathrm{mg} / \mathrm{Kg}$. b.w. & $12.23^{*}$ \\
Immunosuppressed treated with URMeOH $200 \mathrm{mg} / \mathrm{Kg}$ b.w. &
\end{tabular}


Neutrophils phagocytose microorganisms that are sequestered and destroyed in phagocytic vacuoles, phagolysosomes, by the oxidative action of activated species (derived from the activity of NADPH oxidase, NO synthase, and MPO) and the action of granulocyte proteolytic enzymes (Babior 2000). NADPH oxidase is activated very rapidly in the plasma membrane and in specific granules (Karlsson et al. 2002). The fusion of the specific granules with the azurophilic granules containing the MPO is at the origin of new activated species, in particular by the action of the MPO whose activity intensifies with the acidification of the medium.

MPO is active on a wide variety of microorganisms that it attacks by oxidation at different points and in different ways (viruses, bacteria, fungi, protozoa) (Babior 2000; Klebanoff 2005). Its bactericidal activity includes a step of attachment to the bacterial membrane at the many points where there are polysaccharide structures, resistant to protease and hydrolytic enzymes.

According to the results obtained experimentally, we can say that the URMeOH extract was able to eliminate Cryptosporidium oocysts in the stools and intestinal sections in the infected immunocompetent and immunosuppressed groups treated with URMeOH for two weeks representing the number of Cryptosporidium oocysts were decreased in all other subgroups which means that the extract has a preventive effect against parasitic infection. Rats treated with the extract showed a decrease in the MPO value compared to the pathological which means that the metabolites adjusted the activity of the enzyme.

\section{ACKNOWLEDGEMENTS}

We would like to extend our gratitude and appreciation to Laura Rinaldi, Professore associato di Parassitologia e Malattie Parassitarie presso il Dipartimento di Medicina Veterinaria e Produzioni Animali, Università degli Studi di Napoli Federico II., Naple (Italy) for the technical assistance to the carrying out this important work.

\section{REFERENCES}

Adetumbi M, Javor GT, Lau BHS.1986. Allium sativum (garlic) inhibits lipid synthesis by Candida albicans. Antimicrob Agents Chemother 30: 499-501.

Atanasov AG, Waltenberger B, Pferschy-Wenzig EM, Linder T.2015. Discovery and resupply of pharmacologically active plant-derived natural products: A review.Biotechnol Adv 33 (8): 1582-1614.

Babior BM. 2000. Phagocytes and oxidative stress. Amer J Med 109 (1): 33-44.

Benhouda A, Yahia M, Benhouda D, Bousnane $\mathrm{N}$ et al.2014. Antimicrobial and antioxidant activities of various extracts of
Hyoscyamus albus L. and Umbilicus rupestris L. leaves Algerian. J Nat Prod 2: 1 4-17.

Benhouda A. and Yahia M. 2015. Toxicity and anti-inflammatory effects of methanolic extract of Umbilicus rupestris L. leaves (Crassulaceae). Int J Pharm Bio Sci 6 (1): 395-408.

Bouzid M, Hunter PR, Chalmers RM, Tyler KM. 2013. Cryptosporidium pathogenicity and virulence. Clin Microbiol Rev 26 (1): 115-134.

Camenga DL, Nathanson N, Cole GA. 1974. Cyclophosphamidepotentiated West Nile viral encephalitis: relative influence of cellular and humoral factors. J Inf Dis130: 634-641.

Casemore DP. 1991. Laboratory methods for diagnosing cryptosporidiosis. J Clin Pathol 44 (6): 445-451.

Di Genova BM and Tonelli RR. 2016. Infection strategies of intestinal parasite pathogens and host cell responses. Front Microbiol 7: 1-16.

Farthing MJ. 2000. Clinical aspects of human cryptosporidiosis. Contrib. Microbiol 6: 50-74.

Fayer R, Morgan U, Upton SJ. 2000. Epidemiology of Cryptosporidium: Transmission, detection and identification. Intl J Parasitol 30 (12-13): 1305-1322.

Gaafar MR. 2007. Effect of solar disinfection on viability of intestinal protozoa in drinking water. J Egypt Soc Parasitol 37 (1): 65-86.

Gaafar MR.2012. Efficacy of Allium sativum (garlic) against experimental cryptosporidiosis. Alexandria J Med 48 (1): 59-66.

Gonzalez-Tejero RM, Casares-Porcel M, Sanchez-Rojas PC, RamiroGutierrez MJ, et al.2008.Medicinal plants in the Mediterranean area: Synthesis of the results of the project Rubia. J Ethnopharmacol 1 (16): 341-357.

Karlsson A, Dahlgren C. 2002. Assembly and activation of the neutrophil NADPH oxidase in granule membranes. Antioxid. Redox Signal 4: 49-60.

Khan AA, Rahmani AH, Aldebasi YH, Aly SH. 2014. Biochemical and pathological studies on peroxidases-An updated review. Glob. J. Health Sci 6: 87-98.

Khan AA, Alsahli MA, Rahmani AH. 2018. Myeloperoxidase as an active disease biomarker: recent biochemical and pathological perspectives. Med Sci (Basel) 6 (2): 33.

Klebanoff SJ. 2005. Myeloperoxidase: friend and foe. J Leukocyte Biology 77 (5): 598-625.

Leitch GJ and He Q. 2001. Cryptosporidiosis-an overview. J Biomed Res25 (1): 1-16.

Lumb R, Lanser JA, O’Donoghue PJ. 1998. Electrophoretic and immunoblot analysis of Cryptosporidium oocysts. Immunol Cell Biol 66: 369-370.

Molan AL, Duncan AJ, Barry TN, McNabb WC.2003. Effects of condensed tannins and crude sesquiterpene lactones extracted from chicory on the motility of larvae of deer lungworm and gastrointestinal nematodes. Parasitol Int 52 (3): 209-18.

Nicholls SJ, Hazen SL. 2005. Myeloperoxidase and cardiovascular disease. Arterioscler Thromb Vasc Biol 25: 1102-1111.

Serteyn D, Grulke S, franck T. 2003. La myéloperoxydase des neutrophiles, une enzyme de défense aux capacités oxydantes. Ann Méd Vét 147: 79-93.

Sparks H, Nair G, Castellanos-Gonzalez A. 2015. Treatment of Cryptosporidium: what we know, gaps, and the way forward. Curr Trop Med Rep 2 (3): 181-187.

Sponseller JK, Griffiths JK, Tzipori S. 2014. The evolution of respiratory cryptosporidiosis: evidence for transmission by inhalation. Clin Microbiol Rev 27 (3): 575-586.

Williams JE. 1998. Diagnostic medical parasitology. Parasitol Today 14 (3): 125-6.

Yamaguchi R, Kawata J, Yamamoto T, Ishimaru Y, Sakamoto A, Ono T, Narahara S, Sugiuchi H, Hirose E, Yamaguchi YY. 2015. Mechanism of interferon- $\gamma$ production by monocytes stimulated with myeloperoxidase and neutrophil extracellular traps. Blood Cells Mol Dis 55: 127-133. 